\title{
Zinco no plasma e eritrócito de atletas profissionais de uma equipe feminina

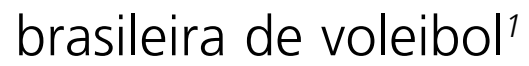

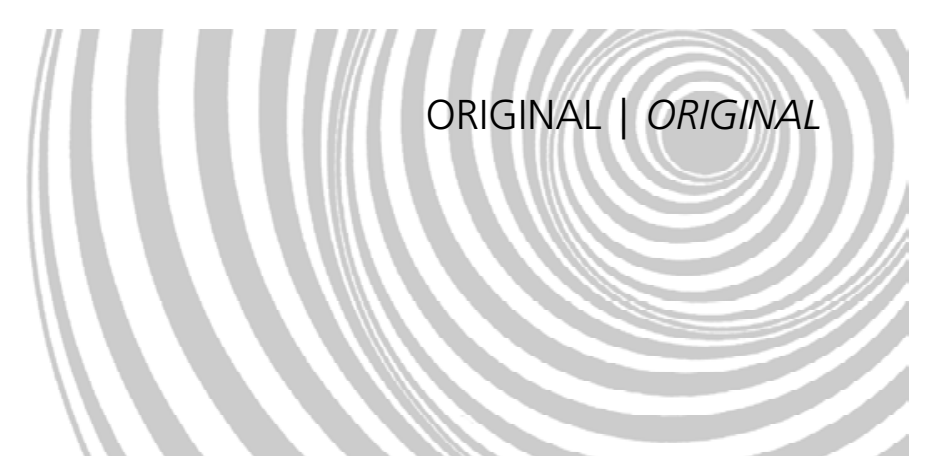

\author{
Plasma and erythrocyte zinc in Brazilian \\ professional female volleyball players
}

Louise Farah SALIBA ${ }^{2}$

Vera Lúcia Cardoso Garcia TRAMONTE²

Gerson Luis FACCIN ${ }^{3}$

\section{RESU M O}

\section{Objetivo}

Avaliar o estado nutricional relativo ao zinco de 12 atletas profissionais do voleibol feminino.

\section{Métodos}

Determinou-se a concentração de zinco plasmático e eritrocitário por espectrofotometria de absorção atômica, e avaliou-se a ingestão dietética por meio de registro alimentar de três dias, de 12 atletas profissionais do sexo feminino, integrantes de uma equipe de voleibol de Curitiba, PR, na faixa etária de 18 a 24 anos, no ano de 2003.

\section{Resultados}

Todas as atletas apresentaram valores normais de zinco plasmático. Quanto ao zinco eritrocitário, 100,00\% apresentaram valores abaixo da normalidade. Em relação à ingestão dietética de zinco, 83,33\% apresentaram adequação. Decréscimos no conteúdo de zinco eritrocitário podem representar o efeito agudo de exercícios prolongados pela utilização enzimática da superóxido dismutase ou anidrase carbônica.

\section{Conclusão}

O estado nutricional das atletas relativo ao zinco parece estar inadequado, pois, apesar dos valores normais para a concentração de zinco plasmático, a concentração eritrocitária esteve abaixo da faixa de normalidade para todas as atletas.

Termos de indexação: avaliação dietética; esporte; estado nutricional; zinco.

\footnotetext{
1 Artigo elaborado a partir da dissertação de L.F. SALIBA, intitulada "Estado nutricional relativo a ferro e zinco de atletas profissionais de uma equipe feminina brasileira de voleibol." Programa de Pós-Graduação em Nutrição, Universidade Federal de Santa Catarina; 2003.

2 Programa de Pós-Graduação em Nutrição, Centro de Ciências da Saúde, Universidade Federal de Santa Catarina. Caixa Postal 476, Bloco J, $4^{\circ}$ andar, 88040-900, Florianópolis, SC, Brasil. Correspondência para/Correspondence to: L.F. SALIBA. E-mail:<lou.farah@yahoo.com.br>.

3 Laboratório de Nutrição Experimental, Departamento de Nutrição, Centro de Ciências da Saúde, Universidade Federal de Santa Catarina. Florianópolis, SC, Brasil.
} 


\section{A B S T R A C T}

\section{Objective}

This study aimed to evaluate the nutritional status of zinc in 12 female volleyball players.

\section{Methods}

Zinc levels in plasma and erythrocytes were assessed by atomic absorption spectrophotometry. Zinc intake of 12 professional female athletes of a volleyball team from Curitiba, PR, aging from 18 to 24 years in 2003, was determined by a 3-day food record.

\section{Results}

All athletes presented normal plasma zinc values. Erythrocyte zinc was lower than normal in $100 \%$ of the athletes. Dietary intake of zinc was adequate in $83.33 \%$. The significant decreases in erythrocyte content could represent the acute effects of prolonged exercises which increases superoxide dismutase or carbonic anhydrase utilization.

\section{Conclusion}

The athletes' zinc nutritional status seemed to be inadequate. They had normal plasma zinc levels but the erythrocyte zinc was below the normal values for all of them.

Indexing terms: zinc; nutritional status; nutrition assessment; sports.

\section{N T R O D U ÇÃ O}

Atletas profissionais podem estar submetidos a demandas especiais, devido ao processo metabólico aumentado, resultante do exercício intensivo e treino regular, que podem também aumentar as necessidades de micronutrientes, tanto pelo aumento das taxas de degradação como pelo aumento das perdas corpóreas. Esse fator, o descanso inadequado e a ingestão de pouca energia, em combinação, podem comprometer o sistema imune e o expor a infecções e lesões ${ }^{1}$.

Muitas atletas femininas, particularmente aquelas que participam de esportes que enfatizam a magreza, têm ingestão sub-ótima de energia e nutrientes e estão em risco de deficiência de micronutrientes e de comprometimento do estado nutricional, incluindo fadiga, desidratação, retardo no crescimento e imunocompetência prejudica$\mathrm{da}^{2-5}$. O aumento do esforço físico, decorrente do exercício, e a inadequação dietética expõem-nas a distúrbios orgânicos de todas as ordens ${ }^{6}$.

Entre os micronutrientes, o zinco é um dos de maior importância para o metabolismo humano. Quanto às funções biológicas do zinco, consideradas as diferentes espécies, existem mais de 200 metaloenzimas que dependem dele, seja estruturalmente ou para atividade catalítica, tais como anidrase carbônica, carboxipeptidase A, superóxido dismutase (SOD), álcool desidrogenase, fosfatase alcalina, timidina quinase, DNA polimerase, RNA polimerase, as quais participam da síntese e degradação do ácido nucléico e do metabolismo de carboidratos, lipídeos e proteínas ${ }^{7,8}$.

Esse elemento traço tem impacto sobre os mediadores da imunidade, tais como enzimas, peptídeos tímicos e citocinas, explicando a grande importância do estado nutricional de zinco na regulação da ativação, proliferação e apoptose das células linfóides 9 .

Além das funções supracitadas, algumas enzimas contendo zinco, como a anidrase carbônica e a lactato desidrogenase, estão envolvidas no metabolismo intermediário durante o exercício ${ }^{10}$.

É importante ressaltar que o zinco está entre os nutrientes essenciais para a função normal do sistema antioxidante endógeno ${ }^{11}$. Existem algumas evidências de que o exercício aumenta a produção de radicais livres. Tem sido estimado que $4 \%$ a $5 \%$ do oxigênio consumido durante a respiração não é completamente reduzido a água 
e, ao invés disso, forma radicais livres. Portanto, como o consumo de oxigênio aumenta durante $o$ exercício, um aumento concomitante ocorre na produção de radicais livres e peroxidação lipídica ${ }^{12}$.

É conhecido que o exercício pode mudar agudamente as concentrações de zinco circulante. Porém, a base para a aparente interação adversa entre a atividade física e o zinco circulante não é clara $^{10}$. Há várias razões possíveis para baixas concentrações do zinco plasmático em atletas, incluindo a baixa ingestão pela dieta, a perda excessiva durante os exercícios, a expansão do volume plasmático durante o treino, que dilui a concentração de zinco, e a redistribuição do zinco plasmático para outros tecidos ${ }^{13}$.

O diagnóstico do estado nutricional de zinco não é fácil. Um grande número de indicadores tem sido proposto, mas eles oferecem dificuldades a serem consideradas e/ou interpretadas $^{14,15}$. Apesar da importância desse mineral e do crescente conhecimento sobre suas funções, ainda não há um indicador único, que seja aceito universalmente, apropriado para avaliar com precisão o estado nutricional relativo ao zinco de um indivíduo ${ }^{16}$.

O zinco plasmático é, atualmente, o biomarcador mais aceito e utilizado do estado nutricional relativo ao zinco, apesar de sua especificidade imperfeita e da baixa sensibilidade ${ }^{17}$. Ainda que o zinco plasmático seja um critério válido do estado nutricional de zinco, ele por si só é inadequado, pois outros fatores fisiológicos afetam as suas concentrações ${ }^{18}$. Entre as diversas medidas bioquímicas que têm sido relacionadas com o estado nutricional relativo ao zinco em humanos, está a concentração de zinco no eritrócito ${ }^{19}$. Vários pesquisadores têm sugerido que o zinco nas células sangüíneas, sejam leucócitos ou eritrócitos, pode refletir o teor de zinco tecidual mais precisamente ${ }^{20}$. O zinco encontrado nos eritrócitos não reflete mudanças recentes nos níveis de zinco orgânico de um indivíduo, e é um parâmetro de estado nutricional relativo ao zinco de prazo mais longo 21 .
No que se refere ao zinco, a avaliação do estado nutricional compreende medidas de consumo alimentar, concentrações de zinco plasmático, eritrocitário, urinário e indicadores funcionais, como a análise da atividade de metaloenzimas: anidrase carbônica, fosfatase alcalina e carboxipeptidases ${ }^{22}$.

Nesse sentido, a partir da relevância desse mineral no metabolismo humano e em especial para atletas, e sabendo-se que há uma lacuna de pesquisas com atletas brasileiros e parâmetros nutricionais, o objetivo deste trabalho foi avaliar o estado nutricional relativo ao zinco no plasma e no eritrócito de atletas profissionais de uma equipe feminina brasileira de voleibol.

\section{MÉ T O D OS}

O estudo foi realizado com 12 atletas profissionais do sexo feminino, integrantes de uma equipe de voleibol, na faixa etária de 18 a 24 anos, no mês de fevereiro, durante a Superliga de Vôlei Feminino. A competição ocorreu entre novembro de 2003 e maio de 2004 , sendo que as equipes que apresentaram melhor desempenho participaram durante toda a temporada. Embora a competição tenha se iniciado ao final de 2003, os treinamentos começaram em julho de 2003.

As atletas perfaziam um tempo médio de treino de $4 \mathrm{~h} 30 \mathrm{~min}$ por dia, sendo dividido em dois períodos, pela manhã e à tarde. As folgas ocorriam aos sábados à tarde e aos domingos. Esses dois períodos subdividiam-se entre treino de preparo físico, musculação (uma hora), aeróbio (30 minutos), e o restante treino com bola (em quadra).

Neste estudo se buscou descrever o estado nutricional de uma equipe profissional. Dessa maneira, por se caracterizar como um estudo não-experimental e descritivo, optou-se por uma seleção não-probabilística e intencional dos participantes.

Este estudo foi aprovado pelo Comitê de Ética em Pesquisa com Seres Humanos da Uni- 
versidade Federal de Santa Catarina, sob o número 016/2003. Após receberem as informações pertinentes ao estudo, as atletas que concordaram em participar assinaram um termo de consentimento livre e esclarecido.

O sangue foi coletado com as atletas em jejum alimentar de $12 \mathrm{~h}$ e com repouso de exercício físico de 36h, visando aumentar a confiabilidade dos resultados.

Para avaliação do estado nutricional relativo ao zinco, foram coletados $10 \mathrm{ml}$ de sangue venoso de cada atleta, utilizando-se seringas plásticas com agulhas em aço inoxidável, descartáveis, transferidos logo após a tubos de centrífuga desmineralizados, os quais continham anticoagulante citrato de sódio a 30\% (100 $\mathrm{\mu l} / 10 \mathrm{ml}$ sangue). As amostras foram transportadas e acondicionadas em isopor com gelo, para o Laboratório de Hematologia do Departamento de Patologia Médica da Universidade Federal do Paraná, para separação das frações do sangue para análise do zinco no plasma e no eritrócito.

As amostras foram centrifugadas a 3000 x g (HERMLE Z 323K) por 15 minutos, em uma temperatura de $4^{\circ} \mathrm{C}$, para a separação do plasma. Para a coleta deste, o sobrenadante foi aspirado e acondicionado em tubos Eppendorf de $1,5 \mathrm{ml}$ previamente desmineralizados. As amostras foram congeladas para análises, que foram feitas no dia seguinte à coleta.

A preparação do eritrócito para análise do zinco foi realizada com a lavagem da papa de hemáceas obtida do sangue total, com $5 \mathrm{ml}$ de solução salina isotônica a 0,9\% e subseqüente homogeneização lenta por inversão. Após esse procedimento, o material foi centrifugado por 10 minutos em uma centrífuga a $4^{\circ} \mathrm{C}$, em uma velocidade de 10000xg (HERMLE Z323K). Quando necessário, foi equilibrado o peso dos tubos no rotor com a própria solução salina. Ao término da centrifugação, foi aspirado o sobrenadante. Após aspirar a solução salina, repetiu-se o procedimento de lavagem das papas à centrífuga por mais duas vezes. Ao final da última centrifugação, a massa de eritrócitos foi cuidadosamente ex- traída, sem encostar no fundo do tubo, inserindo uma micropipeta no centro. O material coletado foi acondicionado em 3 Eppendorfs desmineralizados por amostra, e congelado imediatamente após.

As amostras de plasma e zinco congeladas foram transportadas, acondicionadas em isopor com gelo, via aérea, no mesmo dia da coleta, para o Laboratório de Nutrição - Minerais da Faculdade de Ciências Farmacêuticas da Universidade de São Paulo, onde foram analisadas as concentrações de zinco no plasma e no eritrócito.

\section{Parâmetros bioquímicos de avaliação do zinco}

\section{Determinação de zinco no plasma}

A determinação da concentração de zinco no plasma foi feita por espectrofotometria de absorção atômica com aparelho Hitachi Modelo Z - 5000 equipado com lâmpada de catodo oco, calibrado com comprimento de onda de $213,9 \mathrm{~nm}$ e leitura em duplicata, fenda de $0,4 \mathrm{~nm}$, chama oxidante com mistura de ar (15,0l/min): acetileno (2,0l/min).

Para fazer a curva padrão de calibração foi utilizado o padrão Tritisol ${ }^{\otimes}$ (MERCK), com as seguintes concentrações: 0,$05 ; 0,10 ; 0,20 ; 0,30$; 0,50 e 1,00 $\mu \mathrm{g} / \mathrm{ml}$. O padrão foi diluído em água Milli ${ }^{\circledR}$ com $3 \%$ de glicerol (para igualar viscosidade entre padrão e amostra) e $1 \%$ de ácido nítrico.

Foram preparadas duas alíquotas de cada amostra de plasma, por meio de diluição em água Milli Q ${ }^{\circledR}$ na proporção 1:5, e aspiradas diretamente na chama do aparelho.

Os resultados calculados a partir das absorbâncias obtidas representam a média das leituras em duplicatas e das concentrações das amostras preparadas em duplicatas, e foram expressos em $\mu \mathrm{g} \mathrm{Zn/dl}$. Os valores considerados normais para o zinco plasmático foram $>75 \mu \mathrm{g} / \mathrm{d}^{23}$. Valores abaixo deste foram considerados deficiência de zinco no plasma. 


\section{Determinação de zinco no eritrócito}

Para a determinação de zinco no eritrócito foi utilizado o mesmo equipamento, tendo-se adotado as mesmas condições da determinação de zinco no plasma.

Para fazer a curva padrão de calibração foi utilizado o padrão Tritisol ${ }^{\circledR}$ (MERCK), com as seguintes concentrações: 0,$10 ; 0,20 ; 0,30 ; 0,50$ e 1,00 $\mu \mathrm{g} / \mathrm{ml}$. O padrão foi diluído em água Milli $Q^{\circledR}$ com $1 \%$ de ácido nítrico.

Foram realizadas duas diluições dos eritrócitos para a dosagem do zinco no eritrócito, expressa em termos de massa de zinco por massa de hemoglobina. Na primeira diluição, denominada lisado 1, foi adicionado $900 \mu l$ da água Milli $Q^{\circledR}$ a $300 \mu l$ da amostra. Nesse lisado foi determinada a dosagem da hemoglobina, para posterior correção no cálculo do zinco no eritrócito ( $\mu \mathrm{g} \mathrm{Zn/g} \mathrm{hemoglobina).} \mathrm{Na} \mathrm{segunda} \mathrm{diluição,}$ denominada lisado 2, uma alíquota de 200 $\mu$ do lisado 1 foi diluída em $1800 \mu$ l de água Milli Q ${ }^{\circledR}$. Nesse lisado, a concentração de zinco foi determinada por meio de leitura direta no espectrofotômetro de absorção atômica.

A dosagem da concentração de hemoglobina foi realizada pelo método da cianometahemoglobina. Para a dosagem desta, foi diluída uma alíquota de $20 \mu \mathrm{l}$ do lisado $1 \mathrm{em} 5 \mathrm{ml}$ do Reagente de Cor (reativo de Drabkin) Labtest Cat. 43 e realizada leitura direta no espectrofotômetro UV visível Hitachi, modelo U1100, em comprimento de onda de 540nm. O valor da hemoglobina em $\mathrm{g} / \mathrm{dl}$ foi calculado utilizando-se o fator de calibração obtido como o Padrão de Hemoglobina - Labtest Cat. 47.

Os resultados de zinco no eritrócito foram expressos em $\mu \mathrm{g} \mathrm{Zn} / \mathrm{g}$ de hemoglobina, representando a média das leituras das amostras em triplicatas, tanto para o zinco quanto para a hemoglobina.

Os níveis considerados normais de zinco no eritrócito foram de 40 a $44 \mu \mathrm{g} Z n$ por grama de hemoglobina $(\mu \mathrm{g} \mathrm{Zn/g} \mathrm{Hb})^{24}$. Resultados abaixo do valor inferior foram considerados deficiência de zinco no eritrócito.

\section{Avaliação da ingestão de zinco}

A ingestão alimentar de zinco foi estimada utilizando-se registros alimentares de três dias consecutivos, referentes aos alimentos consumidos em dois dias da semana e um de fim de semana. Os dados provenientes dos registros foram analisados utilizando-se um software de nutrição com análise dietética DietPRO ${ }^{\circledR}$, versão $4.0^{25}$, para quantificação de zinco na dieta. Os alimentos anotados nos registros alimentares e que não estavam cadastrados no banco de dados do software foram computados conforme informações dos valores nutricionais expostos nos rótulos dos mesmos. Para a análise e comparação dos resultados, trabalhou-se com a média dos resultados das análises do registro alimentar de três dias fornecida pelo programa.

A verificação da adequação da ingestão de zinco pelas atletas foi feita conforme o Institute of Medicine ${ }^{26}$. Foi prefixado um nível de confiança $\geq 95 \%$ para considerar a dieta adequada ${ }^{27}$.

Os dados registrados foram transportados para o programa SPSS ${ }^{\circledR}$, versão $10.0^{28}$, no qual foram efetuadas as análises estatísticas. As variáveis analisadas permitiram a utilização dos procedimentos descritivos da estatística paramétrica: média, desvio-padrão, valor máximo e valor mínimo. Utilizou-se a Correlação de Spearmann $\left(r^{5}\right)$ para verificar possíveis associações entre as variáveis de estudo (nível de significância de $p \leq 0,05$ ).

\section{RESULTADOS}

Os resultados obtidos da determinação de zinco plasmático e no eritrócito das atletas encontram-se na Tabela 1. Os resultados das análises de zinco no plasma das atletas apresentaram valores acima do ponto de corte. Porém, em relação ao zinco no eritrócito, todos os valores estavam abaixo da faixa de normalidade. Mesmo a atleta 06 , que apresentou o maior valor de zinco 
no plasma, não conseguiu atingir o valor de normalidade para zinco no eritrócito. Apenas uma atleta apresentou resultado maior que $30 \mu \mathrm{g} \mathrm{Zn/g}$ $\mathrm{Hb}$ no eritrócito, as demais atletas obtiveram valores entre 18,55 e $29,12 \mu \mathrm{g} \mathrm{Zn/g} \mathrm{Hb}$ no eritrócito, ou seja, muito abaixo dos valores ideais.

Não foi encontrada correlação entre o zinco no plasma e no eritrócito $\left(r^{s}=-0,021\right.$ e $\left.p=0,948\right)$.

É possível verificar que a média de zinco no eritrócito ficou muito abaixo do valor de referência e que os resultados de zinco no plasma apresentaram maior variação, quando comparados aos do zinco no eritrócito, com desvios-padrão de $13,71 \mu \mathrm{g} / \mathrm{dl}$ e $3,6 \mu \mathrm{g} \mathrm{Zn/g} \mathrm{Hb}$, respectivamente (Tabela 2).

Tabela 1. Concentração de zinco no plasma ( $\mu \mathrm{g} / \mathrm{dl})$ e no eritrócito ( $\mu \mathrm{g} \mathrm{Zn/g} \mathrm{Hb)} \mathrm{de} \mathrm{atletas} \mathrm{profissionais} \mathrm{de} \mathrm{voleibol} \mathrm{femi-}$ nino. Curitiba, 2003

\begin{tabular}{ccc}
\hline Atleta & Zn no plasma $(\mu \mathrm{g} / \mathrm{dl})$ & Zn no eritrócito $(\mu \mathrm{g} \mathrm{Zn/g} \mathrm{Hb})$ \\
\hline 1 & 114,38 & $\mathbf{2 5 , 4 2}$ \\
2 & 92,78 & $\mathbf{2 2 , 8 5}$ \\
3 & 86,20 & $\mathbf{3 0 , 2 9}$ \\
4 & 104,03 & $\mathbf{2 6 , 2 9}$ \\
5 & 80,90 & $\mathbf{2 4 , 3 7}$ \\
6 & 125,10 & $\mathbf{2 9 , 1 2}$ \\
7 & 105,75 & $\mathbf{2 2 , 4 6}$ \\
8 & 96,98 & $\mathbf{2 4 , 6 9}$ \\
9 & 84,68 & $\mathbf{2 6 , 8 3}$ \\
10 & 107,40 & $\mathbf{1 8 , 5 5}$ \\
11 & 93,83 & $\mathbf{1 8 , 6 2}$ \\
12 & 82,95 & $\mathbf{2 3 , 4 4}$ \\
DP & 13,71 & 3,6 \\
\hline
\end{tabular}

Nota: Valores normais de zinco no plasma: $>75 \mu \mathrm{g} / \mathrm{d}^{23}$; Valores normais de zinco no eritrócito: 40 e $44 \mu \mathrm{g} \mathrm{Zn/g} \mathrm{Hb}{ }^{24}$; Resultados em negrito $=$ abaixo do ponto de corte.
Na Figura 1 podem-se visualizar as variações dos valores de zinco no plasma $(\mu \mathrm{g} / \mathrm{dL}$ ) encontrados das atletas participantes do estudo em relação ao ponto de corte de deficiência em zinco. Observa-se que as atletas 5 e 12 foram as que apresentaram valores mais próximos do ponto de corte de deficiência de zinco plasmático, 80,90 e $82,95 \mu \mathrm{g} / \mathrm{dll}$, respectivamente, apesar de estarem normais.

A Figura 2 apresenta as variações nas concentrações de zinco no eritrócito das atletas e caracteriza como sub-ótimo o estado nutricional relativo ao zinco das mesmas. Apesar da variação, todas elas apresentaram resultados bem abaixo da faixa de normalidade.

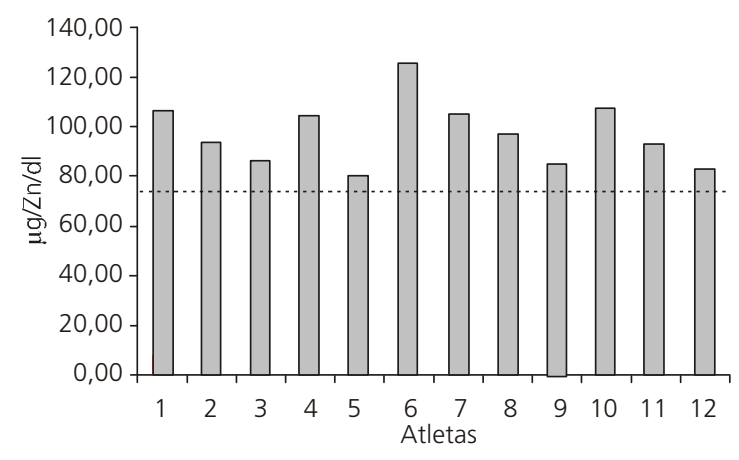

Figura 1. Concentração de zinco no plasma ( $\mu \mathrm{g} / \mathrm{dl})$ em relação ao ponto de corte de deficiência de zinco plasmático, $<75 \mu \mathrm{g} / \mathrm{dl}$, de atletas profissionais de voleibol feminino. Curitiba, 2003.

Tabela 2. Médias, medianas, desvios-padrão, valores mínimos e máximos da concentração de zinco no plasma ( $\mu \mathrm{g} / \mathrm{dl}$ ) e no eritrócito ( $\mu \mathrm{g} \mathrm{Zn} / \mathrm{g} \mathrm{Hb}$ ) de atletas profissionais de voleibol feminino. Curitiba, 2003.

\begin{tabular}{lccccc}
\hline Variáveis & Média & Mediana & Desvio-padrão & Mínimo & Máximo \\
\hline Zn plasma $(\mu \mathrm{g} / \mathrm{dl})$ & 97,92 & 95,41 & 13,71 & 80,90 & 125,10 \\
Zn eritrócito $(\mu \mathrm{g} \mathrm{Zn/g} \mathrm{Hb})$ & 24,41 & 24,53 & 3,60 & 18,55 & 30,29 \\
\hline
\end{tabular}

Nota: Valores normais de zinco no plasma: $75 \mu \mathrm{g} / \mathrm{dl}^{23}$; Valores normais de zinco no eritrócito: 40 e $44 \mu \mathrm{g} \mathrm{Zn} / \mathrm{g} \mathrm{Hb}{ }^{24}$; Resultados em negrito= abaixo do ponto de corte. 


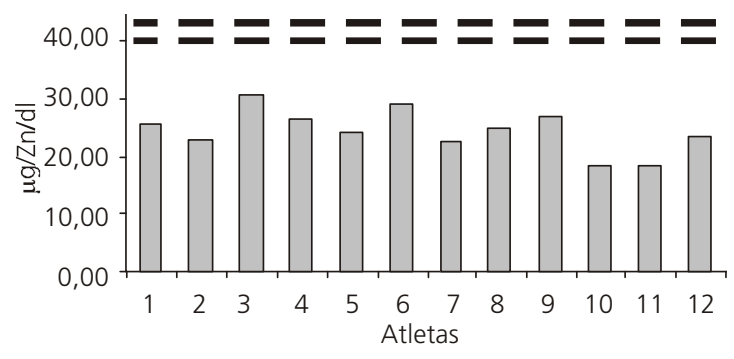

Figura 2. Concentração de zinco no eritrócito ( $\mu \mathrm{g} \mathrm{Zn/g} \mathrm{Hb)} \mathrm{em}$ relação à faixa de normalidade, 40 e $44 \mu \mathrm{g} \mathrm{Zn} / \mathrm{g} \mathrm{Hb}$, de atletas profissionais de voleibol feminino. Curitiba, 2003.

Em relação à avaliação da adequação do consumo alimentar de zinco, entre as 12 atletas, $10(83,33 \%)$ apresentaram o consumo adequado desse mineral. Para as demais atletas, não necessariamente o consumo estava inadequado, mas, conforme avaliação, houve uma probabilidade menor de o consumo estar adequado $70,00 \%$ para $1(8,33 \%)$ das atletas e $30,00 \%$ para $1(8,33 \%)$.

\section{DISCUSSÃO}

Neste estudo, encontrou-se que 100\% das atletas apresentaram valores normais de zinco plasmático e que $100 \%$ delas apresentaram baixo teor de zinco nos eritrócitos, com valores abaixo do limite normal.

Lukaski et al. ${ }^{29}$, avaliando o zinco plasmático em 29 atletas e em um grupo controle de 28 indivíduos na pré e pós-temporada, e Gleeson et al. ${ }^{30}$, em 8 indivíduos não treinados, antes e após treinamento, encontraram valores normais, corroborando os achados do presente estudo com as atletas do vôlei, em que $100 \%$ delas apresentaram valores acima do ponto de corte para deficiência de zinco.

Porém, é válido ressaltar que alterações nas concentrações de zinco plasmático podem ser afetadas por diversos fatores tais como flutuações em horas do dia, estado nutricional, a quantidade e o tipo de alimento ingerido, entre outros ${ }^{31}$. Conforme Lukaski10, uma explicação para a interação entre a atividade física e o zinco circulante é que o decréscimo de zinco circulante está associado à ingestão inadequada deste mineral. Quando o suprimento de zinco dietético é muito baixo ou se uma ingestão marginal é consumida por um longo período de tempo, os ajustes homeostáticos podem não ser suficientes para repor as perdas do mineral e ocorre um balanço de zinco negativo ${ }^{32}$.

Em relação ao presente estudo com as atletas de voleibol, a maior parte delas (83,33\%) apresentou consumo adequado de zinco. As demais atletas podem ter consumido quantidades de zinco que atenderiam suas necessidades. Provavelmente, devido a estes resultados, não houve deficiência de zinco plasmático. A resposta orgânica à ingestão de zinco foi demonstrada em um estudo com mulheres deficientes marginalmente nesse mineral, que receberam suplementação e apresentaram aumento nos níveis plasmáticos ${ }^{33}$.

Por outro lado, como uma conseqüência de sua regulação e outros fatores comuns que podem influenciar sua distribuição, os níveis de zinco plasmático não são considerados um reflexo preciso da ingestão dietética ou do estado nutricional relativo ao zinco ${ }^{16}$. Segundo Nuviala et al. ${ }^{34}$, em um estudo realizado com mulheres de diversas modalidades esportivas, os níveis séricos e a ingestão de zinco não parecem estar relacionados.

Além da dieta, outro fator a ser considerado é a característica mista do vôlei (resistência + força), uma vez que em esportes aeróbios ocorre maior utilização de zinco plasmático. Tuya et al. ${ }^{35}$, em um estudo realizado com atletas masculinos (modalidades aeróbias e anaeróbias), observaram que os de modalidade anaeróbia apresentavam maiores concentrações de zinco plasmático, em comparação com os de modalidade aeróbia. Segundo os autores, essa razão pode estar relacionada com as funções do zinco como cofator em diversas enzimas que agem no metabolismo energético, na imunidade e como antioxidante, e são mais relevantes em esportes com um componente aeróbio significante. Isso poderia explicar 
as baixas concentrações em atletas de modalidades aeróbias, os quais mobilizariam grandes quantidades de zinco para suportar as funções metabólicas e aumentar a SOD intracelular. Koury et al. ${ }^{36}$ Observaram, em seu estudo realizado com atletas de elite de diferentes modalidades, que os de modalidades aeróbias de longa distância e de alto impacto apresentaram índices de proteção antioxidante maiores do que daqueles das modalidades de baixo impacto e curta distância, sugerindo que existe uma adaptação da capacidade antioxidante ao treinamento específico.

Por outro lado, considerando que as atletas de vôlei feminino são submetidas a um treinamento intenso, inclusive corrida, além do treinamento em quadra, poderia ocorrer uma utilização moderada de zinco para suprir as necessidades metabólicas impostas pelo exercício. Essa deman$\mathrm{da}$, associada com as perdas via suor, poderia levar à depleção de zinco, interferindo nas concentrações de zinco no eritrócito. A perda de minerais pela sudorese durante o treinamento e a competição, principalmente quando realizados em elevadas temperaturas, é substancial ${ }^{37}$. Poder-se-ia ponderar que seria uma explicação para os valores 100\% abaixo para o zinco no eritrócito. Segundo Micheletti et al. ${ }^{11}$, um decréscimo significante no conteúdo de zinco no eritrócito representa o efeito agudo de exercícios prolongados.

Outra hipótese que poderia justificar os valores deficientes de zinco no eritrócito seria a de que o exercício pode redistribuir o zinco do plasma ao eritrócito e a outros tecidos corporais e vice-versa, conforme revisão feita por Beals \& Manore ${ }^{2}$ e Singh et al. ${ }^{38}$, no estudo sobre o estado nutricional de atletas com desordens alimentares subclínicas.

Singh et al.$^{38}$ avaliaram zinco plasmático e eritrocitário de corredoras e não corredoras. Os valores encontrados estavam abaixo do ponto de corte de zinco plasmático para algumas corredoras, diferentes dos achados das atletas de vôlei, e os níveis de zinco no eritrócito estavam normais, porém mais altos, quando comparados valores de corredoras e não corredoras. Os resultados de zinco plasmático e eritrocitário desse estudo, comparando corredoras e não corredoras, e os encontrados com as atletas de vôlei, sugerem diferenças entre modalidades, aeróbia (corrida) e mista (voleibol).

Ainda em relação aos resultados de zinco no eritrócito das atletas do presente estudo, poder-se-ia cogitar um eventual quadro de desnutrição pregressa, uma vez que existe um período de latência para a manifestação da deficiência de um mineral. Em geral, são necessários vários meses para os sinais da deficiência se tornarem aparentes, principalmente quando a ingestão é marginalmente baixa ${ }^{2}$. Porém, neste estudo não houve correlação entre a ingestão de zinco e seus níveis plasmáticos nem eritrocitários, além de que, considerando os dados da análise sobre o consumo alimentar de zinco, a maior parte das atletas apresentou ingestão adequada deste nutriente.

Outra hipótese seria que a depleção do zinco no eritrócito das atletas de vôlei poderia ser causada pela sua utilização na anidrase carbônica, que tem sua atividade aumentada durante o exercício físico. O zinco incorporado na anidrase carbônica contribui para a maior parte do zinco eritrocitário ${ }^{17}$. A anidrase carbônica catalisa a conversão de $\mathrm{CO}_{2}$ e $\mathrm{H}_{2} \mathrm{O}$ a $\left[\mathrm{HCO}_{3}\right]$, assim como a reação reversa. Essa reação é lenta e pouco dióxido de carbono seria carreado se não fosse pela ação da anidrase carbônica ${ }^{39}$. Tem sido sugerido que o controle dessa reação durante o exercício pode ser mediado por alterações na concentração de zinco no eritrócito ${ }^{40}$. Como o exercício físico aumenta a respiração em 10 a 15 vezes e, conseqüentemente, aumenta o volume excretado de $\mathrm{CO}_{2}$, aumentaria a utilização de zinco presente no eritrócito, podendo, então, se pensar na explicação para os valores tão baixos encontrados de zinco no eritrócito nas atletas de vôlei.

\section{O N CLUS Ã O}

O estado nutricional das atletas relativo ao zinco parece estar inadequado, pois apesar de 
terem apresentado valores normais para a concentração de zinco no plasma, a concentração de zinco no eritrócito esteve abaixo da faixa de normalidade para todas as atletas.

Dificuldades são encontradas para a avaliação do estado nutricional de zinco em humanos, em função da ausência de um método definitivo que o avalie com precisão. Além disso, os trabalhos realizados com a dosagem de zinco no eritrócito são escassos, ainda mais com avaliações em atletas e, principalmente, atletas de voleibol ou de modalidades esportivas com componentes mistos. Este fato dificulta comparações e interpretações de resultados, apontando para a necessidade de mais estudos nesta área.

\section{A GR ADECIMENTO}

Ao Prof. Dr. Rodrigo Siqueira Reis, pela análise estatística.

\section{REFERÊ NCIAS}

1. Venkatraman JT, Leddy J, Pendergast D. Dietary fats and immune status in athletes: clinical implications. Med Sci Sports Exerc. 2000; 32(Suppl 7):389-95.

2. Beals KA, Manore MM. Nutritional status of female athletes with subclinical eating disorders. J Am Diet Assoc. 1998; 98(4):419-25.

3. American College of Sports Medicine. Joint statement position: Nutrition and athletic performance. Med Sci Sports Exerc. 2000; 32(12): 2130-45.

4. Montero A, Lopez-Varela S, Nova E, Marcos A. The implication of the binomial nutrition-imunity on sportswomen's health. Eur J Clin Nutr. 2002; 56(Suppl 3):38-41.

5. Papadopoulou SK, Papadopoulou SD, Gallos CK. Macro and micronutrient intake of adolescent Greek female volleyball players. Int J Sport Nutr Exerc Metab. 2002; 12(1):73-80.

6. Ribeiro BG, Soares EA. Avaliação do estado nutricional de atletas de ginástica olímpica do Rio de Janeiro e São Paulo. Rev Nutr. 2002; 15(2):181-91.

7. Fairweather-Tait SJ. Zinc in human nutrition. Nutr Res Rev. 1988; 1:23-37.
8. Prasad AS. Discovery of human zinc deficiency and studies in an experimental human model. Am J Clin Nutr. 1991; 53(2):403-12.

9. Dardenne M. Zinc and imune function. Eur J Clin Nutr. 2002; 56(Suppl 3):20-3.

10. Lukaski HC. Magnesium, zinc, and chromium nutriture and physical activity. Am J Clin Nutr. 2000; 72(2 Suppl):585-93.

11. Micheletti A, Rossi R, Rufini S. Zinc status in athletes: relation to diet and exercise. Sports Med. 2001; 31(8):577-82.

12. Clarkson PM, Thompson H. Antioxidants: what role do they play in physical activity and health? Am J Clin Nutr. 2000; 72(Suppl):637-46.

13. Haymes EM. Minerais de traço e exercícios. In: Wolinski I, Hickson JF. Nutrição no exercício e no esporte. São Paulo: Roca; 1996.

14. Hambidge M. Human zinc deficiency. J Nutr. 2000; 130(Suppl):1344-9.

15. Portela ML, Weisstaub AR. Basal Urinary Zinc/ Creatinine Ratio as an Indicator of Dietary Zinc Intake in Healthy Adult Women. J Am Coll Nutr. 2000; 19(3):413-7.

16. Wood RJ. Assessment of marginal zinc status in humans. J Nutr. 2000; 130(Suppl):1350-4.

17. Hambidge M. Biomarkers of trace mineral intake and status. J Nutr. 2003; 133(3 Suppl):948-55.

18. O'Dell BL. Role of zinc in plasma membrane function. J Nutr. 2000; 130(5 Suppl):1432-6.

19. Kenney MA, Ritchey SJ, Culley P, Sandoval W, Moak $S$, Schilling P. Erythrocyte and dietary zinc in adolescent females. Am J Clin Nutr. 1984; 39(3):446-51.

20. Davis CD, Milne DB, Nielsen FH. Changes in dietary zinc and copper affect zinc-status indicators of postmenopausal women, notably, extracellular superoxide dismutase and amyloid precursor proteins. Am J Clin Nutr. 2000; 71(3):781-8.

21. Denise $M$, Sílvia MFC. Importância do zinco na nutrição humana. Rev Nutr. 2004; 17(1):79-87.

22. Marreiro DN, Fisberg M, Cozzolino SMF. Considerações sobre o estado nutricional relativo ao zinco na obesidade. Cad Nutr. 1998; 16(3): 31-40.

23. lyengar $\mathrm{V}$, Woittiez J. Trace elements in humans clinical specimens: evaluation of literature data to identify reference values. Clin Chem Lab Med. 1988; 34(3):474-81.

24. Guthrie HA, Picciano MF. Human nutrition. Boston: McGraw Hill; 1994. 654p.

25. DietPRO. Software de avaliação e prescrição de dieta [computer program]. Versão 4.0. Viçosa, MG: Agromídia Software Ltda; 2003. 
26. Institute of Medicine; Food and Nutrition Board Dietary reference intakes for vitamin A, Vitamin $\mathrm{K}$ arsenic, boron, chromium, copper, iodine, iron, manganese, molybdenum, nickel, silicon, vanadium, and zinc. The National Academies Press; 2002 [cited 2003 Jun 23]. Available from: http://books.nap.edu/books

27. Marchioni DML, Slater B, Fisberg RM. Aplicação das Dietary Reference Intakes na avaliação da ingestão de nutrientes para indivíduos. Rev Nut. 2004; 17(2):207-16.

28. Statistical Package for Social Sciences [computer program]. Versão 10.0 for windows. Chicago, Illinois; 1999.

29. Lukaski HC, Hoverson BS, Gallagher SK, Bolonchuk WW. Physical training and copper, iron, and zinc status of swimmers. Am J Clin Nutr. 1990; 51(6):1093-9.

30. Gleeson M, Walsh NP, Blannin AK, Robson PJ, Cook $L$, Connelly $A E$, et al. The effect of severe eccentric exercise-induced muscle damage on plasma elastase, glutamine and zinc concentrations. Eur J Appl Physiol. 1998; 77(6):543-6.

31. Schumann K, Classen HG, Hages M, PrinzLangenohl R, Pietrzik K, Biesalski HK. Bioavailability of oral vitamins, minerals, and trace elements in perspective. Arzneimittelforschung. 1997; 47(4): 369-80.

32. King JC, Shames DM, Woodhouse LR. Zinc homeostasis in humans. J Nutr. 2000; 130(Suppl): 1360-66.

33. Donangelo CM, Woodhouse LR, King SM, Viteri FE, King JC. Supplemental zinc lowers measures of iron status in young women with low iron reserves. J Nutr. 2002; 132(7):1860-4.

34. Nuviala RJ, Lapieza MG, Bernal E. Magnesium, zinc, and copper status in women involved in different sports. Int J Sport Nutr. 1999; 9(3):295-309.

35. Tuya IR, Gil EP, Mariño MM, Carra RMG-M, Misiego AS. Evaluation of the influence of physical activity on the plasma concentrations of several trace metals. Eur J Appl Physiol. 1996; 73(3-4): 299-303.

36. Koury JC, Oliveira AV Jr, Portella ES, Olilveria CF, Lopes GC, Donangelo CM. Zinc and copper biochemical indices of antioxidant status in elite athletes of different modalities. Int J Sport Nutr Exerc Metab. 2004; 14(3):358-72.

37. Koury JC, Donangelo CM. Zinco, estresse oxidativo e atividade física. Rev Nutr. 2003; 16(4):433-41.

38. Singh A, Deuster PA, Moser PB. Zinc and copper status in women by physical activity and menstrual status. J Sports Med Phys Fitness. 1990; 30(1): 29-36.

39. McArdle WD, Katch FI, Katch VL. Permuta e transporte dos gases. In: Fisiologia do exercício. Energia, nutrição e desempenho humano. Rio de Janeiro: Guanabara Koogan; 1998.

40. Mundie TG, Hare B. Effects of resistance exercise on plasma, erythrocyte, and urine $\mathrm{Zn}$. Biol Trace Elem Res. 2001; 79(1):23-8.

Recebido em: 25/6/2005

Versão final reapresentada em: 13/2/2006 Aprovado em: 19/5/2006 